\title{
Generalized Framework of OKID for Linear State-Space Model Identification
}

\author{
Francesco Vicario, Minh Q. Phan, Richard W. Longman, and Raimondo Betti
}

\begin{abstract}
This paper presents a generalization of observer/Kalman filter identification (OKID). OKID is a method for the simultaneous identification of a linear dynamical system and the associated Kalman filter from input-output measurements corrupted by noise. OKID was originally developed at NASA as the OKID/ERA algorithm. Recent work showed that ERA is not the only way to complete the OKID process and paved the way to the generalization of OKID as an approach to linear system identification. As opposed to other approaches, OKID is explicitly formulated via state observers providing an intuitive interpretation from a control theory perspective. The extension of the OKID framework to more complex identification problems, including nonlinear systems, is also discussed.
\end{abstract}

\section{Introduction}

The goal of system identification is to find a mathematical model of a dynamic system from measured input and output data. Over the last decades, it has found many applications in many areas of engineering, for example in control systems, where the system model is needed for controller design, and structural health monitoring, where the structure model is monitored over time to detect damage. More generally, system identification plays a crucial role wherever a mathematical model of a dynamic system is needed. State-space models are particularly appealing since they

F. Vicario

Philips Research North America, Briarcliff Manor, NY 10510, USA,

e-mail: francesco.vicario@philips.com (at Columbia University when this work was done)

R. W. Longman, R. Betti

Columbia University, New York, NY 10027, USA,

e-mail: rw14@columbia.edu, rb68@columbia.edu

M. Q. Phan

Dartmouth College, Hanover, NH, 03755, USA, e-mail: minh.q.phan@ dartmouth.edu 
lend themselves to analysis via linear algebra techniques, robust numerical integration, and modern control design methods.

Many researchers have developed algorithms for state-space model identification. Connections between the available methods is a complex task and the lack of a general theory for system identification represents a barrier when an engineer or a researcher attempts to enter the field. Many of the available algorithms have been explained in a general framework known as subspace identification (SID) [10]. However, SID does not encompass all the available methods. Additionally, the SID framework is purely based on linear algebra, which makes the derivation and description of the resulting algorithms lack intuition and interpretation.

One of the algorithms that do not belong to the SID family is OKID/ERA (observer/Kalman filter identification followed by eigensystem realization algorithm) $[1,5]$. OKID/ERA was originally developed at NASA for the purpose of identifying lightly-damped structures [4]. Nevertheless it is applicable to any linear system and as such it found countless applications. The strength of OKID/ERA is that, under the standard assumptions of Kalman filter theory (zero-mean and white process and measurement noise), it optimally handles the noise inevitably present in the data. In contrast, other algorithms for system identification do not address noise (hence they are referred to as deterministic algorithms) and the model they identify generally results biased. Recently it was demonstrated how ERA is not the only way to complete the OKID process. More specifically, ERA can be replaced by any deterministic algorithm for state-space model identification $[12,15]$.

In the light of these recent findings, this paper shows how OKID can now be viewed as a general approach to linear system identification. The OKID framework explains many algorithms for state-space model identification, in a way similar to what SID does for other algorithms. In contrast to SID, OKID lends itself to an intuitive interpretation in terms of state observers, which is emphasized in this paper. Extension of OKID to more complex problems such as output-only and nonlinear system identification is briefly discussed in the last section.

\section{Problem Statement}

Consider the following linear-time-invariant (LTI) system in state-space form

$$
\begin{aligned}
x(k+1) & =A x(k)+B u(k)+w_{p}(k) \\
y(k) & =C x(k)+D u(k)+w_{m}(k)
\end{aligned}
$$

where $x \in \mathbb{R}^{n \times 1}$ is the state vector, $u \in \mathbb{R}^{m \times 1}$ is the input vector, $y \in \mathbb{R}^{q \times 1}$ is the output vector, $A \in \mathbb{R}^{n \times n}$ is the system matrix, $B \in \mathbb{R}^{n \times m}$ is the input matrix, $C \in \mathbb{R}^{q \times n}$ is the output matrix, and $D \in \mathbb{R}^{q \times m}$ is the direct influence matrix. Additionally, the vectors $w_{p} \in \mathbb{R}^{n \times 1}$ and $w_{m} \in \mathbb{R}^{q \times 1}$ represent the zero-mean white process and measurement noise, with covariance matrices $Q \in \mathbb{R}^{n \times n}$ and 
$R \in \mathbb{R}^{q \times q}$, respectively. They are uncorrelated with $u$ and $y$ but they can be mutually correlated with cross-covariance matrix $S \in \mathbb{R}^{n \times q}$.

The objective is to identify the system in Eq. (1), i.e., find the matrices $A, B$, $C$, and $D$, from a dataset of input-output measurements. The data are assumed to be of sufficient length and richness so that the system in Eq. (1) can be correctly identified. Neither the noise sequences $w_{p}$ and $w_{m}$ nor their covariance matrices $Q$, $R$, and $S$ are known.

It would be ideal to extract from the measured input-output data also the gain of the optimal linear observer of the system state, to be used for instance to implement state-feedback control laws. Note that the optimal observer gain is uniquely determined by the system and noise covariance matrices. Whereas the measurement noise covariance can usually be estimated via dedicated experiments on the sensors, the process noise covariance is harder to assess. Extracting the gain directly from measured input-output data is then particularly useful.

\section{State Observers}

At the core of OKID is the concept of state observer. More specifically, OKID relies on the existence of an optimal LTI one-step-ahead state predictor. In the presence of zero-mean, white process and measurement noise, such an optimal observer is the well-known steady-state Kalman filter. It is worth pointing out that in this paper the term steady-state will be often omitted for brevity.

\subsection{Linear-Time-Invariant (LTI) Observers}

A typical problem in system dynamics and control theory is the estimation of the state $x$, given the system matrices $A, B, C, D$ and past input-output measurements. Indeed, in most cases the state is not measured and only a subset (or, more generally, a linear combination) of the state variables is measured as output.

The state estimation problem for the LTI system in Eq. (1) can be formulated as follows. Given the system model and the measured values of the input $u$ and output $y$ from time sample 0 to $k$, what is the best estimate $\hat{x}$ that we can get for the state $x$ at the next time step $k+1$ ? Among all the possible state estimators (or state observers), OKID makes use of LTI observers.

The most general form for a LTI state state observer is

$$
\begin{aligned}
\hat{x}(k+1) & =F \hat{x}(k)+H u(k)+G y(k) \\
\hat{y}(k) & =C \hat{x}(k)+D u(k)
\end{aligned}
$$

where $F \in \mathbb{R}^{n \times n}, H \in \mathbb{R}^{n \times m}$ and $G \in \mathbb{R}^{n \times q}$. Equation (2b) is added to provide an estimate of the true system output as well. Such an estimate is indicated by $\hat{y}$, which 
is also known as the observer output. Note that Eq. (2) is the equation of a dynamic system, whose state is driven by $u$ and $y$, which are the input and output of the original system in Eq. (1) but from the point of view of the observer are both inputs. In other words, an observer is a dynamical system itself, whose state is $\hat{x}$.

It is well known that in the presence of zero-mean white noise, the Kalman filter is the optimal linear observer for the system in Eq. (1). Optimality in this context is defined as minimization of the second moment of the state estimation error at every time step $k$. The matrices of the Kalman filter are generally time-varying. However, after the initial transient and under the assumption of stationary noise, i.e., noise with constant mean and covariance, the Kalman filter matrices become constant. At steady state, the Kalman filter is then in the form of Eq. (2). The steady-state Kalman filter is the optimal LTI observer.

The Kalman filter matrices can be derived in several ways and, remarkably, from different starting points as there are a few properties that uniquely characterize it. For examples, [1] defines the Kalman filter as the linear observer minimizing the second moment of the state estimation error, i.e., $\mathbb{E}\left[(x(k)-\hat{x}(k))^{\mathrm{T}}(x(k)-\hat{x}(k))\right]$. Among the other properties that uniquely define the Kalman filter, there are a few related to the so-called observer output residuals

$$
\varepsilon(k)=y(k)-\hat{y}(k)
$$

The Kalman filter output residuals are a zero-mean, white random process, with variance minimized with respect to any other LTI observer and they are orthogonal to past input-output data.

\subsection{Kalman Filter in Predictor Form}

$$
\begin{aligned}
\hat{x}(k+1) & =\bar{A} \hat{x}(k)+\bar{B} v_{x}(k) \\
\hat{y}(k) & =C \hat{x}(k)+D u(k)
\end{aligned}
$$

where $\bar{B}=[B-K D K]$ and $v_{x}(k)=\left[u^{\mathrm{T}}(k) y^{\mathrm{T}}(k)\right]^{\mathrm{T}}$. The predictor form expresses the Kalman filter as a state-space model with input given by the measured system input and output ( $u$ and $y$ ). Note that the dynamics of Eq. (4) is governed by $\bar{A}=$ $A-K C$ instead of $A$, hence it is sometimes called bar form. 


\subsection{Kalman Filter in Innovation Form}

$$
\begin{aligned}
\hat{x}(k+1) & =A \hat{x}(k)+B^{\prime} v_{x}^{\prime}(k) \\
\hat{y}(k) & =C \hat{x}(k)+D u(k)
\end{aligned}
$$

where $B^{\prime}=[B K]$ and $v_{x}^{\prime}(k)=\left[u^{\mathrm{T}}(k) \varepsilon^{\mathrm{T}}(k)\right]^{\mathrm{T}}$. The innovation form of the Kalman filter is driven by inputs different from those in Eq. (4), i.e., $u$ and $\varepsilon$ instead of $u$ and $y . \varepsilon$ is also known as the innovation process, hence the name innovation form. Even more importantly, the dynamics of Eqs. (4) and (5) are governed by different matrices $(\bar{A}$ and $A)$. Nevertheless, the two forms of the Kalman filter are equivalent in the sense that both provide the same time histories for $\hat{x}$ and $\hat{y}$. It is worth noting also how the state-space model in Eq. (5) explicitly includes the matrices $A, B, C$, and $D$ of the original system to be identified.

\subsection{Deadbeat Observer}

In the absence of noise in Eq. (1), there exists a LTI observer that, at steady state, has zero state estimation error. Such an observer is obviously the optimal observer in the absence of noise and is called deadbeat because it converges to the exact state after exactly $n$ time steps (if the system is single-output; more generally, a number of steps equal to the smallest integer greater than or equal to $n / q$ is needed). A deadbeat observer is characterized by $\bar{A}$ having all the eigenvalues at the origin. As a consequence, $\bar{A}^{p}=0$ for $p \geq n / q$.

\section{Observer/Kalman Filter Identification}

As will be explained in section 5 and shown in Fig. 1, the OKID approach is made of two main steps: first the solution of an equation that is the same for all the OKIDbased algorithms and then the identification of the observer used to derive such an equation. The second step can be implemented in several different ways, giving rise to many methods belonging to the same OKID framework. More details about derivations, proofs, algorithms and examples can be found in [11].

\subsection{OKID Equation}

The main difficulty in state-space model identification is that, although the model in Eq. (1) is linear, from the viewpoint of system identification both the state $x$ and the 
matrices $A$ and $C$ are unknowns. Their products in Eq. (1) make the identification problem nonlinear. OKID relies on an observer to remove such nonlinearity.

Defining $L=[H G]$ and $v_{x}(k)=\left[u^{\mathrm{T}}(k) y^{\mathrm{T}}(k)\right]^{\mathrm{T}}$ the most general form of LTI observer, Eq. (2), becomes

$$
\begin{aligned}
\hat{x}(k+1) & =F \hat{x}(k)+L v_{x}(k) \\
\hat{y}(k) & =C \hat{x}(k)+D u(k))
\end{aligned}
$$

Note the similarity with Eq. (4). Equation (6) is an observer in predictor form.

Propagating Eq. (6a) forward in time by $p-1$ time steps and then shifting the time index backward by $p$, we obtain

$$
\hat{x}(k)=F^{p} \hat{x}(k-p)+T z(k)
$$

where $z(k)=\left[v_{x}^{\mathrm{T}}(k-1) v_{x}^{\mathrm{T}}(k-2) \ldots v_{x}^{\mathrm{T}}(k-p)\right]^{\mathrm{T}}$ and $T=\left[I F \ldots F^{p-2} F^{p-1}\right] L$. As will be proven later, the stability of the observer guarantees that $F^{p}$ becomes negligible for sufficiently large values of $p(p>>n)$. Equation (7) yields then the following relation expressing the current state as a function of the sole past input and output values

$$
\hat{x}(k)=T z(k)
$$

Plugging Eq. (8) into Eq. (6b) and recalling the definition of observer output residuals, we obtain

$$
y(k)=\Phi v(k)+\varepsilon(k)
$$

where $v(k)=\left[u^{\mathrm{T}}(k) z^{\mathrm{T}}(k)\right]^{\mathrm{T}}$ and $\Phi=\left[D C L C F L \ldots C F^{p-2} L C F^{p-1} L\right]$. Equation (9) relates the input and output, without the state appearing explicitly. Note that $\Phi$ contains the sequence of Markov parameters of the observer in predictor form. The Markov parameters of a discrete-time linear model correspond to its unit pulse response and they have the property of being unique for a given system. Equation (9) can be written for each time step $k \geq p$ of the measured data record, to obtain the following set of equations in matrix form

$$
Y=\Phi V+E
$$

where

$$
\begin{aligned}
Y & =\left[\begin{array}{llll}
y(p) y(p+1) & \ldots & y(l-1)
\end{array}\right] \\
V & =\left[\begin{array}{llll}
v(p) & v(p+1) & \ldots & v(l-1)
\end{array}\right] \\
E & =\left[\begin{array}{llll}
\varepsilon(p) & \varepsilon(p+1) & \ldots & \varepsilon(l-1)
\end{array}\right]
\end{aligned}
$$

and $l$ is the number of collected time samples. Equation (10) is the OKID core equation. $Y$ and $V$ are known (from measurements), $\Phi$ and $E$ are not. By having a sufficiently long data record, the set of equations in Eq. (10) is overdetermined. Considering $E$ as an error term, it is possible to find the least-squares (LS) 
solution $\tilde{\Phi}=Y V^{\dagger}$, where ${ }^{\dagger}$ denotes the Moore-Penrose pseudoinverse of a matrix. Associated with the LS solution are the LS residuals $\tilde{E}=Y-\tilde{\Phi} V$.

The orthogonality property of the LS residuals establishes a fundamental link between the LS solution to Eq. (10) and the Kalman filter associated with the system to be identified (Eq. (1)) and the statistics $(Q, R, S)$ of the noise embedded in the data. The steady-state Kalman filter is the unique LTI observer whose output residuals are orthogonal to past input-output data. Hence, it can be proven that [11]

1. The LS solution of Eq. (10) is an estimate for the sequence of Markov parameters of the Kalman filter in the form of Eq. (4).

2. The LS residuals of Eq. (10) correspond to (are an estimate of) the output residuals of the Kalman filter.

In other words, by solving by LS Eq. (10), which was derived from the most general form of LTI observer (Eq. (2)), such a LTI observer is made the optimal LTI observer, namely the steady-state Kalman filter. Hence, $\tilde{\Phi}$ is an estimate of

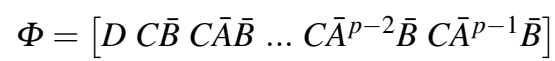

Having established that the observer used in the derivation of Eq. (10) is the Kalman filter, then its stability is guaranteed and the assumption of $F^{p} \approx 0$ for large $p$ to derive Eq. (8) is justified.

\subsection{Identification of the Observer/Kalman Filter}

The Kalman filter at the core of OKID can be identified from its Markov parameter sequence $\Phi$, whose estimate $\tilde{\Phi}$ is obtained from the LS solution of the OKID core equation. As the sequence of Markov parameters of a dynamic system corresponds to its unit pulse response, one can identify the matrices $\bar{A}, \bar{B}, C$, and $D$ of the Kalman filter in predictor form via algorithms such as ERA [2] or ERA-DC [3] capable of extracting the model matrices (also known as realization) from the system unit pulse response. From the so-obtained Kalman filter matrices, $A, B$, and $K$ can be extracted from $\bar{A}$ and $\bar{B}$. Relabeling the matrix blocks in $\bar{B}$ as $\bar{B}=\left[\begin{array}{ll}\bar{B}_{1} & \bar{B}_{2}\end{array}\right]$ where $\bar{B}_{1} \in \mathbb{R}^{n \times m}$ and $\bar{B}_{2} \in \mathbb{R}^{n \times q}$, we can recover

$$
K=\bar{B}_{2} \quad B=\bar{B}_{1}+K D \quad A=\bar{A}+K C
$$

In the original OKID/ERA and OKID/ERA-DC algorithms (Reference [5]), a preliminary operation on the sequence of Markov parameters estimated from Eq. (10) was done. Such an operation can be interpreted as the conversion from the Markov parameters of the Kalman filter in predictor form into the Markov parameters of the Kalman filter in innovation form

$$
\Psi=\left[D C B^{\prime} C A B^{\prime} C A^{2} B^{\prime} \ldots\right]
$$


The traditional OKID/ERA and OKID/ERA-DC algorithms complete then the identification process feeding $\Psi$ to ERA or ERA-DC to extract the matrices $A, B, C, D$, and $K$.

As previously pointed out, the Kalman filter is itself a dynamic system in statespace form. For instance, consider the innovation form of the Kalman filter, Eq. (5), with input given by $u$ and $\varepsilon$ and output by $\hat{y}$. $u$ is known from measurements, $\varepsilon$ and $\hat{y}$ are estimated via the LS solution to the OKID core equation. Both the input and the output of the dynamic system in Eq. (5) are then known. Additionally, in Eq. (5) no (unknown) noise term is present. We have just constructed a new noise-free identification problem: given the time histories of $u, \varepsilon$, and $\hat{y}$, find the matrices $A, B^{\prime}, C$, and $D$. Thanks to the absence of noise, any deterministic system identification method capable of identifying a state-space model from its response to an arbitrary input can be used to solve the new problem. Note that the solution to the new problem is also the solution to the original identification problem. $B$ and $K$ are readily available from $B^{\prime}$.

This gives rise to many OKID-based identification algorithms, as many as the deterministic identification methods from arbitrary input response that one can think of. For instance, one can use algorithms from the subspace family [10], such as deterministic intersection (DI) or deterministic projection (DP), or from the superspace family (SS) [8]. Following the same nomenclature of OKID/ERA and OKID/ERADC, the resulting methods are indicated as OKID/DI, OKID/DP and OKID/SS. The DI, DP and SS methods are considered deterministic because their formulation is based on purely deterministic state-space models, with no process or measurement noise taken into account.

Similar to the innovation form, Eq. (4) also represents a dynamic system whose input $(u$ and $y)$ and output $(\hat{y})$ are known. Any deterministic system identification method can be applied to identify the state-space model in Eq. (4), i.e., the matrices $\bar{A}, \bar{B}, C$, and $D$. From the latter, Eq. (13) allows one to recover also the desired matrices $A, B$, and $K$.

It is worth adding that other algorithms can be devised simply by replacing DI, DP or SS with other deterministic system identification methods. For instance, one could use any of the subspace algorithms in Reference [10].

\section{OKID Framework}

The previous sections highlighted how different methods for system identification can be developed once the OKID core equation is solved by LS. In this section, we provide an overview of the OKID approach, summarizing the main steps and alternatives characterizing this generalized framework for state-space model identification. 


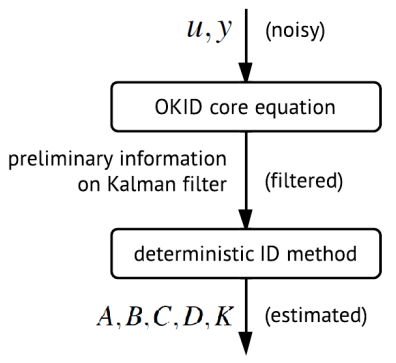

(a)

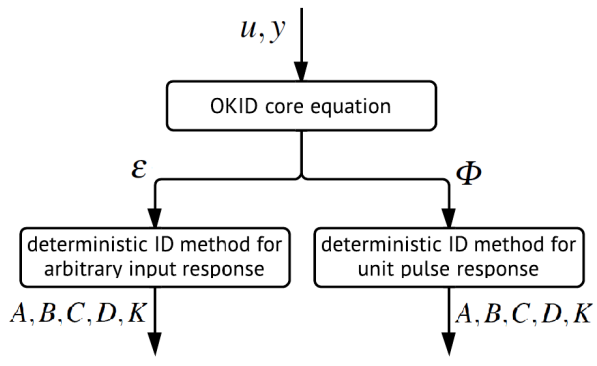

(b)

Fig. 1 OKID as a two-step process (a) and two main approaches within its framework (b).

\subsection{Overview}

All the methods within the OKID framework are made of two main steps (Fig. 1a). First, they find the LS solution to an equation derived via an observer to obtain some information about the optimal observer associated with the system to be identified. Such information is preliminary in the sense that it uniquely characterizes the optimal observer but it is not in a useful model form nor it gives information about the system to be identified. Further processing is needed. The distinctive characteristic of the second step is that it is virtually noise-free. Hence it can be performed with any deterministic state-space model identification method.

Two main alternatives are available for the second step of OKID (Fig. 1b). One is based on the Markov parameters of the optimal observer, the other on the output residuals of the same observer, both estimated in the first step of OKID. The former represent the unit pulse response of the system, hence they can be processed with state-space model identification methods that receive as an input a unit pulse response (e.g., ERA or ERA-DC). On the other hand, using the observer residuals to construct a new deterministic state-space model identification problem requires an identification method capable of processing the response to arbitrary excitation ( $u$ and $\varepsilon$ or $u$ and $y$ are the excitation input to the Kalman filter).

The essence of the second step of OKID is the identification of the optimal LTI observer (steady-state Kalman filter), from which it is then straightforward to recover the system matrices (i.e., the original objective of the identification) thanks to the close relationship between the observer model (Eqs. (4) or (5)) and the system model (Eq. (1)). Regardless of the choice of using observer Markov parameters or output residuals, in the second step of OKID we can decide to identify the Kalman filter in innovation or predictor form. This gives rise to the four branches in the diagram in Fig. 2. Such a diagram is meant to provide the big picture of OKID. Some of the branches further split into different alternatives that are not shown here for brevity and to not get lost into algorithmic details that would hide the main concepts behind the generalized framework of OKID. 


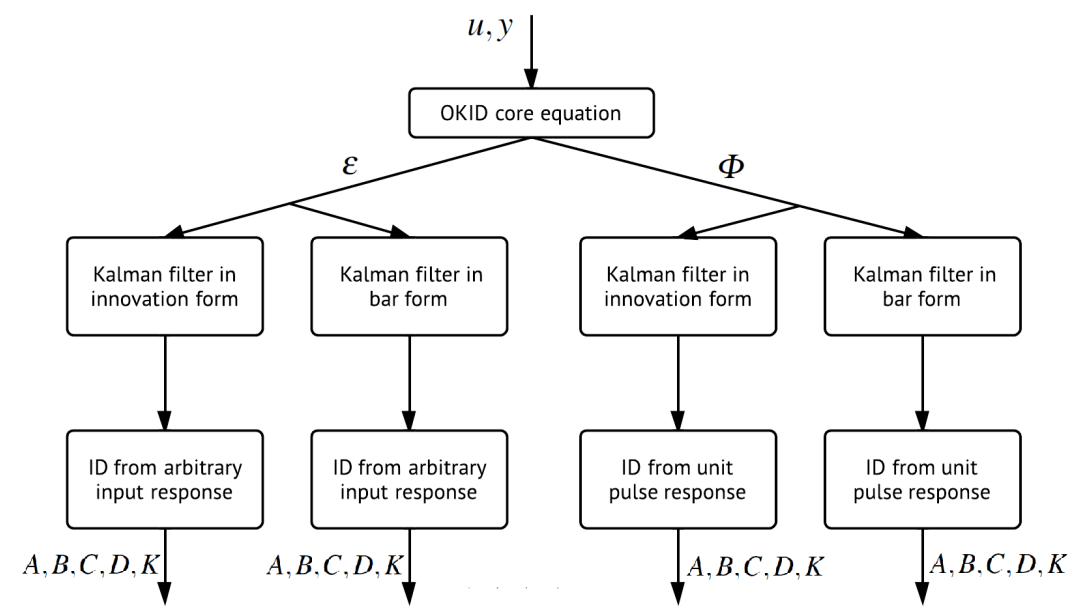

Fig. 2 Four main branches of OKID.

\subsection{Discussion}

To summarize, the core of OKID consists in (i) using a LTI observer to implicitly estimate the true state of the system to be identified, removing the initial nonlinearity of the identification problem; (ii) exploiting the LS solution to guarantee that such an observer is not a random observer with any $F, H$, and $G$ matrices but the optimal LTI observer, whose matrices are closely related to the matrices of the system to be identified. Said link is crucial in OKID. Note how the use of an observer, which eventually turns out to be the optimal LTI observer, has been defined as implicit because the matrices of the observer are not even known before identification. Nevertheless, the sole structure of the LTI observer allows one to derive the OKID core equation, whose LS solution provides some preliminary information on the observer itself.

An elegant and interesting interpretation of OKID in the presence of noise can be found in [15]. The Kalman filter underlying the OKID equation filters out the noise in the measured data rendering the second step of OKID a deterministic system identification problem. As shown in [15], such filtering is generally not perfect because of the error due to the approximation of the OKID core equation $\left(F^{p} \approx 0\right)$, but can be made such via a technique called residual whitening [7]. Note how the OKID core equation can be seen as a converter from stochastic to deterministic system identification. It allows any deterministic system identification method to operate in the second step of OKID in noise-free conditions, as assumed in their formulations. It is also worth noting how the presence of noise prevents direct access to the system model. The associated Kalman filter has to be identified first and then the system model is extracted from the identified Kalman filter model. The Kalman filter is the bridge between noisy data and the system to be identified. 
The Kalman filter can be identified either in innovation or predictor form. However, the form underlying the OKID core equation is the latter. One feature that makes the predictor form special is that the stabilizing properties of the Kalman filter makes $\bar{A}$ more stable than $A$. Hence the number of time steps $p$ necessary to neglect the state dependent term in Eq. (7) is generally smaller than if $A^{p} \hat{x}(k-p)$ were to be neglected. The predictor form is a mechanism to compress the dynamics of the observer. Such compression is maximum in the absence of noise, in which case the optimal steady-state observer is deadbeat and $p$ can be as small as the smallest integer greater than or equal to $n / q$. As a historical note, the basis for the development of OKID was indeed the observation that an extremely efficient compression of the dynamics of a system could be obtained via a deadbeat observer. This was very appealing in the identification of lightly-damped structures, whose $A$ matrix has eigenvalues close to the unit circle and hence its powers decay very slowly (large $p$ ).

\section{Further Extensions}

This paper presented OKID as a general framework for the identification of linear systems from input-output measurements. The same framework has already been successfully extended to more complex problems. The previous sections emphasized how at the core of OKID is the existence of a Kalman filter for the system to be identified. If the input is not measured but can be assumed to be (or approximated as) a white random process, the state-space model of the system is usually written as Eq. (1) without the terms depending on $u$, which are absorbed by $w_{p}$ and $w_{m}$. A Kalman filter exists to optimally estimate the state of such a model that formally has no deterministic input $u$. Hence, OKID can be applied to the identification of systems driven only by unmeasured noise (output-only system identification) with important applications in structural health monitoring [14]. Another development of OKID worth mentioning is in linear-time-varying (LTV) system identification [6].

An even more interesting extension made possible by the generalization of OKID presented in this paper is given by bilinear system identification. Bilinear systems are defined in state-space form like in Eq. (1) but with some additional terms in the state equation involving products between the state variables and the input. Bilinear systems are then a class of nonlinear systems. By proving the existence of optimal bilinear observers with properties similar to the ones of the Kalman filter that led to OKID in the linear case [9], it was possible to develop the first extension of an OKID-based method to nonlinear system identification [13]. This extension is particularly important in the light of the following appealing property of bilinear models. Bilinear models, possibly of high order, can approximate a very broad class of nonlinear systems, namely input-affine systems. At the same time, bilinear models have sufficient mathematical structure to develop techniques for controller and observer design. As a consequence, one can think of bilinear models as a promising universal way to handle nonlinear control problems and bilinear OKID can be seen 
as a general technique for nonlinear system identification. Additionally, following the same approach used to extend OKID to bilinear systems [13], it is expected that OKID-based methods to directly identify some classes of nonlinear systems other than bilinear could be developed. The generalized framework of OKID outlined in this paper holds the promise of new interesting results in nonlinear system identification.

\section{References}

1. Juang, J.-N., Applied System Identification. Prentice Hall, Englewood Cliffs, NJ (1994).

2. Juang, J.-N., Pappa, R.S.: An eigensystem realization algorithm for modal parameter identification and model reduction. Journal of Guidance, Control, and Dynamics, 8(5), 620-627 (1985)

3. Juang, J.-N., Cooper, J.E., Wright, J.R.: An eigensystem realization algorithm using data correlations (ERA/DC) for modal parameter identification. Journal of Control Theory and Advanced Technology, 4(1), 5-14 (1988)

4. Juang, J.-N., Horta, L.G., Phan, M.Q.: System/Observer/Controller/Identification Toolbox. NASA Technical Memorandum 107566 (1992).

5. Juang, J.-N., Phan, M.Q., Horta, L.G., Longman, R.W.: Identification of observer/Kalman filter Markov parameters: theory and experiments. Journal of Guidance, Control, and Dynamics, 16(2), 320-329 (1993)

6. Majji, M., Juang, J.-N., Junkins, J.L.: Observer/Kalman-filter time-varying system identification. Journal of Guidance, Control, and Dynamics, 33(3), 887-900 (2010)

7. Phan, M.Q., Horta, L.G., Juang, J.-N., Longman, R.W.: Improvement of observer/Kalman filter identification (OKID) by residual whitening. Journal of Vibrations and Acoustics, 117, 232-238 (1995)

8. Phan, M.Q., Celik, H.: A superspace method for discrete-time bilinear model identification by interaction matrices. Journal of the Astronautical Sciences, 59(1-2), 421-440 (2012)

9. Phan, M.Q., Vicario, F., Longman, R.W., Betti, R.: Optimal bilinear observers for bilinear state-space models by interaction matrices. International Journal of Control (2015) doi: 10.1080/00207179.2015.1007530

10. Van Overschee, P., De Moor, B., Subspace Identification for Linear Systems: Theory, Implementation, Applications. Ch. 2, Kluwer Academic Publishers, Dordrecht, NL (1996)

11. Vicario, F.: OKID as a general approach to linear and bilinear system identification, $\mathrm{PhD}$ Thesis, Columbia University, New York, NY (2014)

12. Vicario, F., Phan, M.Q., Betti, R., Longman, R.W.: OKID as a unified approach to system identification. Advances in the Astronautical Sciences, 152, 3443-3460 (2014)

13. Vicario, F., Phan, M.Q., Betti, R., Longman, R.W.: Bilinear observer/Kalman filter identification. Advances in the Astronautical Sciences, 152, 1517-1536 (2014)

14. Vicario, F., Phan, M.Q., Betti, R., Longman, R.W.: Output-only observer/Kalman filter identification ( $\mathrm{O}^{3} \mathrm{KID}$ ). Structural Control Health Monitoring, 22(5), 847-872 (2015)

15. Vicario, F., Phan, M.Q., Betti, R., Longman, R.W.: OKID via output residuals: a converter from stochastic to deterministic system identification. Journal of Guidance, Control, and Dynamics (submitted for publication) 